\title{
Adoption of Big Data Technologies for Communication Management in Large Projects
}

\author{
Suhail Memon, Wang Changfeng, Shahid Rasheed, Zulfiqar Hussain Pathan, \\ Sehrish Khan Saddozai, QiuYixin and Liu Yanping; \\ School of Economics and Management, Beijing University of Posts and \\ Telecommunications, China. \\ Suhail_sam06@yahoo.com; wangcf@bupt.edu.cn; shahidrasheed@outlook.com; \\ zulfi2k3bcs@yahoo.com; sehrishkhan5259@gmail.com; 644716494@qq.com; \\ 1162272803@qq.com
}

\begin{abstract}
Big data introduced several novel opportunities for many organizations worldwide. Big data technologies are now available for businesses of all scales and the organizations are adopting them increasingly to capitalize on various business gains. In this era of growing projects globally, big data is not only assisting in information exchange but also bringing convenience for communication management in large scale projects. This study assesses the level of awareness of communication management professionals regarding Big Data technologies and analyzes the contribution of different factors responsible for the rate of adoption of big data technologies for communication management in large projects. The study is based on online surveys and interviews of different organizations and the academia. The findings show that, among others, fear of job loss is a major hindrance while the enhanced information mobility is the major accelerator towards the adoption of big data technologies for communication management. It further establishes that unawareness of the professionals towards big data technologies plays a negative role in their acceptance.
\end{abstract}

Keywords: Communication Management; Big Data Technologies; Large Projects

\section{Introduction}

Big data technologies are concerned with handling the large data sets of large projects containing a variety of data to explore concealed patterns, unidentified correlations, market trends, customer preferences, and precious organizational information sets. According to the literature of Social Sciences Big Data is concerned about differentiation of performance in organizations [1], Barton and Court describe Big Data as an element of competitive advantage for organizations [2]. The growing flexibility of linking asset information with multidimensional attributes of other data is continually being realized by owners of enterprises [3]. Today organizations need technology investments in communication sectors to derive impactful outcomes, flexible solutions, modern IT services, nimble response, and more capable domain control of strategic assets.

Big data integrates technologies to extract a platform to build suitable solutions. Such technologies are being integrated in project based industries to create more agile methods of organizing the communication [4]. In big data we do not see the hardware infrastructure but the user interaction with the applications. Due to the use of internet as communication channel or connecting factor between applications, big data technologies can access any information any time worldwide. With availability of internet connections organizations can communicate globally when conducting large projects. There has been enormous growth in big data technologies in small and medium enterprises as compared 
to larger organizations. The most frequent factors faced by the large organizations have been the untrained IT/communication workforce and controversial views towards Big Data technology usage.

Large projects are set of projects that share high technology, long duration, and more than one firms to work jointly across organizational boundary [5]. The information used for decision making in the management of large projects is produced and stored digitally. Large projects provide huge and compound systems, such as the aircraft experimental facilities, the construction of dams and the national highways and railways. These large projects need to design system integration and communication management as complex system [6]. In these projects multiple firms develop information about complex product system, including various professions and trades, as these firms communicate through the digital system. Digital technologies rapidly transform the project details in between the firms. With adoption of big data technologies the performance of project communication is getting significantly better. Although big data contributes meaningfully in several aspects within large projects, the adoption of big data technologies has been slower than expected because of various reasons including: data security, unawareness, data privacy, and fear of losing control, data protection, bandwidth, and lack of big data business organizations. Figure 1 shows influence of Big Data technologies in large projects.

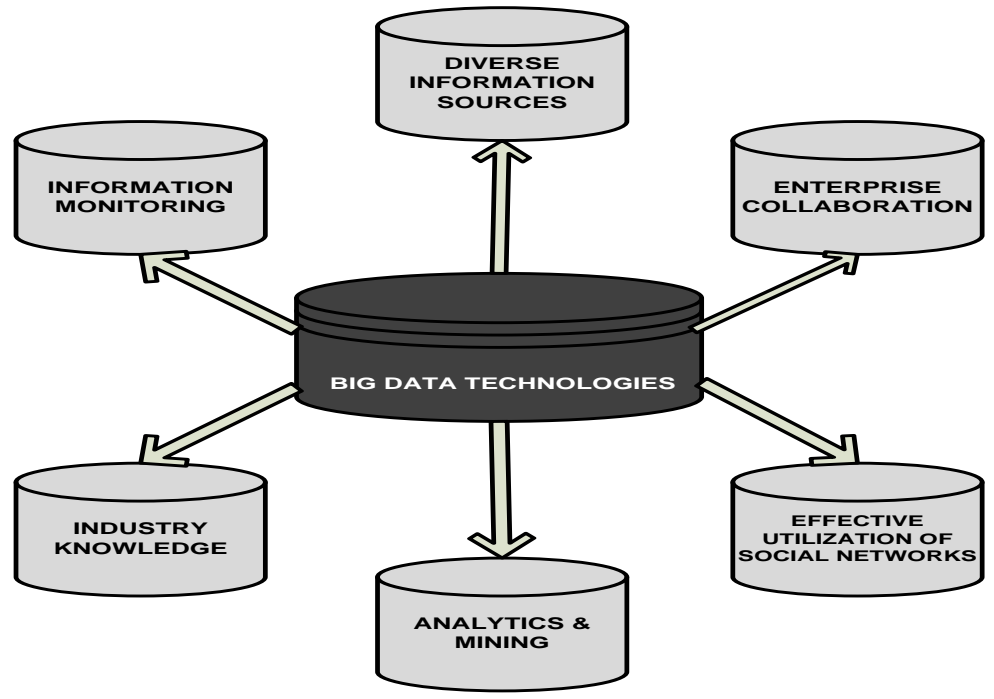

Figure 1. Influence of Big Data Technologies in Large Projects

\section{Communication Management \& Large Projects}

Nowadays information is created as multipurpose as it can be utilized for more than a single functionality [7]. Communication is art of collecting all concerned information, interpreting this information, and efficiently distributing the information to nodes that might need it. Communication has got significant importance to everyone involved in large projects [8]. Proper use of communication and exchange of information maintains stability within projects distributed units. Geographically scattered large projects have become essential practice within advanced organizations because of internationalization of enterprises.

The fast growth of communication technologies has changed the style of collaboration. In this scenario Big Data technologies enable communication across globally spread large projects. Geographically dispersed projects mostly rely on communication media [9], therefore a big challenge for communication management is to establish a relationship between globally distributed project teams [10]. In large projects face-to-face interaction is not always profitable, therefore communication technology provides authenticate 
channels to interact in better ways [11]. The communication hindrances that international projects usually face lean to create missing informal communication, which remains an unremitting struggling reason for globally distributed teams [12].

The infrastructure of one organization that is conducting international projects should be installed with Computer Mediated Communication Systems (CMCS) [13]. These computers are used to exchange and process information, and use telecommunication networks for such exchanges [14]. CMCS include video conferencing, cloud computing, online sharing of information, electronic mails, and Big Data Technologies. The advance technologies that enhance the communication capabilities and provide transition of data and information develop the team work between globally dispersed units of large project. This paper focuses on significant factors regarding Big Data Technologies, which improve the process of communication management during accomplishment of large projects, and the difficulties that organizations are facing during adoption of Big Data technologies.

\section{Identifying and Analyzing the Factors Affecting Adoption of Big Data Technologies in Communication Management}

The management experts and owners of large and small enterprises do know that, communication management workforce are major drivers in adoption of big data technologies in large projects. According to the definition of Big Data technologies, it is the technology for managing, and processing the volumes of structured and unstructured types of information in motion or rest. It implies that information travelling within the networks can be controlled and organized with Big Data technologies. These management capabilities also produce the fear of losing jobs for IT/communication professionals within the enterprises; if the management incorporates these upgrades in the organizational environment then it will become very difficult for these professionals to meet the knowledge levels of Big Data technologies.

The most important cause with respect to slow adoption of Big Data technologies in large projects was the security. Most employees and management officials have argued that using Big Data technologies means storing or delivering information to public networks and customers, and that it will give rise to more chance of hacking and losing information. Securing information has been a major issue and challenge that how big Data technologies are going to be deployed in the IT/ communication Environment. Bandwidth is also a major hindrance to decrease the adoption rate of big data technologies in communication management sector; Bandwidth is considered as transportation channel for Big Data technologies. Majority of the economies are still struggling for the speed of accessing the internet services because they are under developed. The advanced systems and maintenance for good quality of internet are very expensive in these countries and still they are facing such problems. Better environment for big data technologies is directly proportional to high bandwidth of internet services; therefore bandwidth is very important issue for good communication management.

Globally the concept of data privacy exists in variations. Different countries have procedures for maintaining data privacy. If it is known to organizations that using Big Data technologies will not remain protected by country's privacy law then it is not feasible to deploy Big Data technologies in their communication management systems. For this reason many organization depend on personal configured servers. Insufficiency of regulation authorities has a negative impact on the rate of adoption of Big Data technologies all over the world. There are no laws appropriately described through which Big Data technologies are regulated. Big Data Technologies must ensure that their functioning comply with privacy laws, as well as the company's privacy policies and other regulatory requirements. For example organizations that wish to use non public personal information (NPI) must ensure that they are in compliance with Gramm-Leach- 
Bliley Act (GLBA). President Obama also took initiatives for regulations by announcing Big Data Research and Development program. This program was based on six federal departments and authorities to improve the rules and techniques required to organize and glean the technologies of Big Data.

To minimize the risks that may be produced by using Big Data technologies, companies usually elect to de-identify the sharing of information with third party. Application of law presents the potential of companies to use data outside of confines of legislatively developed privacy related boundaries. For example according to the Fair Credit Reporting Act the property of consumer information excludes the information which does not identify individuals similar as aggregate information of blind data. Exceptions to privacy needed de-identified information also exist under HIPAA. The federal trade commission FTC is considering the Big Data issues recently. FTC proposed a program that would initialize greater awareness and control over Big Data technologies. Considering these privacy rules, the developed infrastructures and large scale projects companies would be able to share information across the boundaries.

\section{Methodology and Results}

In a quest to understand the overall awareness of Big Data Technologies among the professionals, the extent of hindrances that impede upon the adoption of Big Data Technologies inside organizations, and the factors that contribute positively towards the acceptance for Big Data Technologies, a series of interviews and surveys were conducted.

The methodology contained face to face interviews and online surveys. A group of communication management professionals from academia and certain professional organizations were interviewed. The resultant of these interviews was supportive to squeeze down the questionnaire. Relatively a big sample of professionals from organization and academia working in different countries of Asia and Europe was selected. Face-to-face interviews helped to fine tune the contents of subject; also the mode of questions became better focused on the adoption of Big Data Technologies in context of communication management in large projects. The random sampling was narrowed to communication management professionals having experience in the field of management with or without the knowledge of big data technologies.

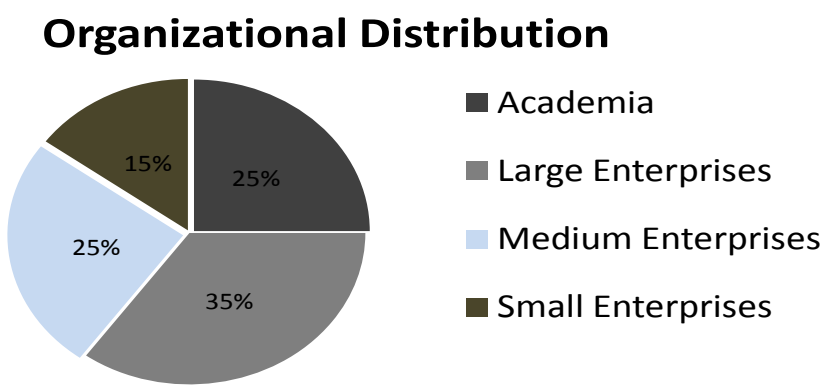

Figure 2. Organizational Distribution for Survey Participants

The academia comprised twenty five percent of the overall sample size, while the rest seventy five percent sample embraced the professional organizations; thirty five percent of which were large enterprises, fifteen percent were medium sized, and the rest belonged to the small enterprises group. Figure 2 shows the organizational distribution that 
participated in the survey. Survey explored three major aspects related to Big Data technologies for communication management: 1) Interviewees Knowledge level; 2) Critical view of communication management professionals aimed growth of Big Data Technologies; 3) Participating elements towards the growth of adoption of Big Data Technologies. The analysis of awareness questionnaire shows that 65 percent of respondents believed in contentious perception that the adoption of Big Data in their organization may harm to their job security. From remaining 35 percent who do not consider it unsecure, 20 percent don't have vivid understanding of Big Data technologies, while the remnant fifteen percent simply think that it is only used for data warehousing. The results made clear reasoning that most of the correspondents are feeling insecure after their organizations have adopted or have intent to accept Big Data technologies. Considering this, it is easy to predict the intensity of this factor as an established hindrance towards acceptance of Big Data Technologies since their early founding.

After analyzing the factors previously categorized as difficulties to the adoption of Big Data technologies in communication management, the questions were made more concise and result oriented. The insufficient regulation authorities and lack of appropriate legislation had been identified as main factor affecting the adoption. The parameters of data security and data protection were also important issues in this process. The expanding number of cell phone devices urged the consideration of information mobility in the mind of correspondents for Big Data technologies. Many people think that, the term information mobility is only connected with smart phones or tablets; but according to modern concept of enterprise, it explains that, how the right information is identified and exchanged across the enterprise; how information is transformed and captured, and how it is used productively to achieve maximum business benefits. Information mobility includes new technologies revolutionizing the way people work together and communicate changes in information management process, as well as work style innovations in a continually developing workforce. Figure 3 captures the analysis of hindrances for adoption of Big Data Technology.

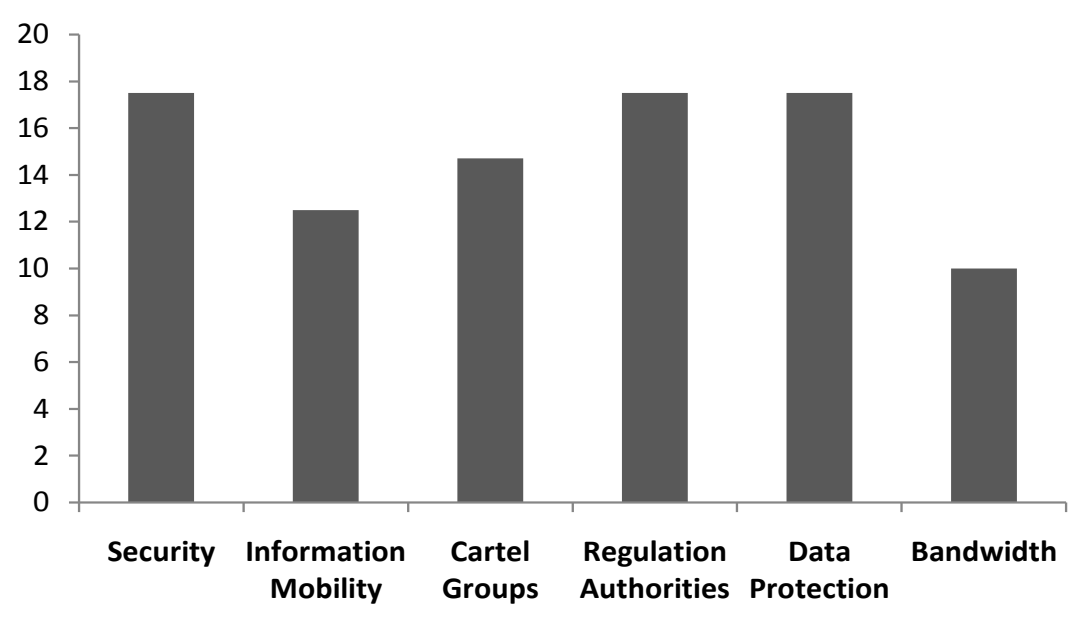

\section{Figure 3. Analysis of Hindrances for adoption of Big Data Technologies}

These correspondents also had concerns regarding cartel groups in the market. Cartel groups are defined as an accord between rival companies to control prices or rule out entry of a fresh competitor in a market. It is a formal company of sellers or buyers that agree to fix procure prices, selling prices, or decrease production using a variety of strategy [15]. Many respondents consider Big Data technologies as storage locations for 
business merely and are still unaware of the depth, functions, and powers they embrace. This exhibits a serious deficit on the awareness part and also shows that organizations are yet to adopt Big Data Technologies in their systems.

Most of the Professionals who respond to the idea of adoption of Big Data in their companies belong to the large enterprises. Majority of Large enterprises which use big data technologies to maximize performance level during large projects, adopt modern and improved techniques as compared to the small or medium enterprises which usually deploy traditional communication technologies and protocols because of limited resources. The correspondents from the organizations those are trying to adopt Big Data technologies don't know exactly when they are actually going to adopt it. The analysis showed that the facts that have helped to increase the adoption rate of Big Data technologies in communication management consisted of six factors: 1) Effective Security 2) Information Mobility 3) Regulation Authorities 4) Utility for Big Data Technologies 5) Reliability 6) Cost Saving. Information Mobility was identified as most prominent element towards maximizing the adoption of Big Data Technologies for communication management in the enterprises. Reliability, feasible cost and utility for Big Data technologies were found equally contributing for adoption rate. Reliability is a feature of any factor (hardware, or software, or a network,) that constantly execute functions according to its disclaimer [16]. It has been deemed amongst one of three interrelated features that must be well thought-out when manufacturing, buying, or using an application or product. Reliability, availability, and serviceability (RAS) are essential facets for development to any system.

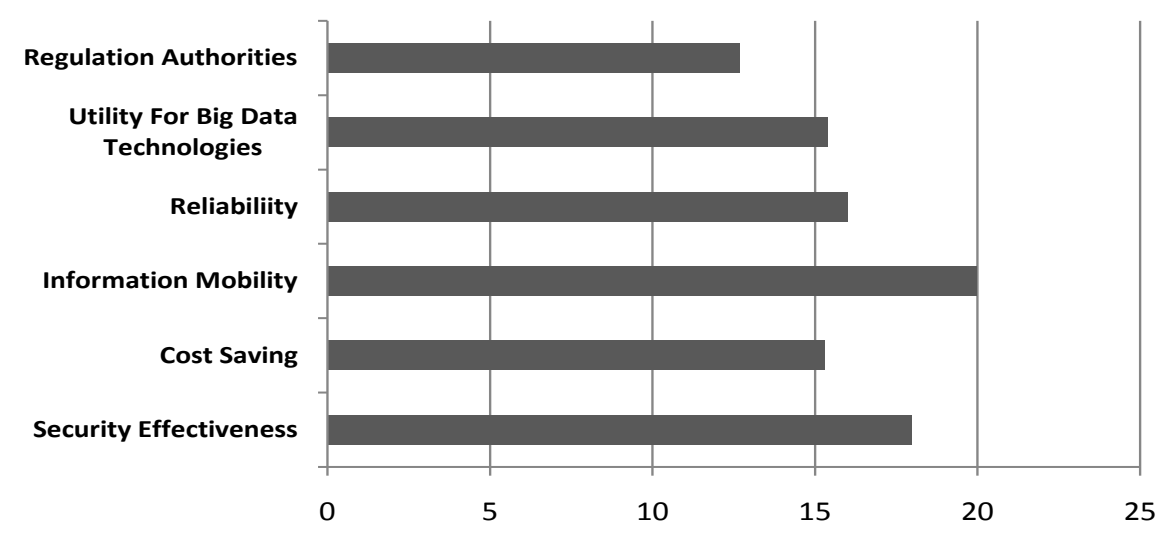

\section{Figure 4. Analysis of Factors that Increase Rate of Adoption for Big Data Technologies}

A reliable service or component is totally free of technological errors; in its mechanism, however, companies often declare quotient of a component's reliability in percentage. The IEEE Reliability society (IEEE RS) persuades mutual effort and share of information between its membership, which includes companies and individuals, connected with all disciplines of engineering, including communication, transportation systems, and aerospace. The factor of regulation authority was considered less contributing during adoption because of slow rate of growth in regulation authorities. Figure 4 shows an analysis of factors which increase adoption rate for Big Data technologies. 
This research measured the perception that communication management professionals should incorporate the Big Data Technologies to get out from the fear of losing job. They should improve their capabilities by using latest updates in communication techniques for productivity and should establish good positions of their companies and enterprises in the competitive environment. This paper also shows that many of professionals need to be educated regarding Big Data Technologies, since as much as twenty percent of the correspondents simply don't know about it. There exists a stringent need to conduct frequent seminars and demonstrations so that communication professionals may be trained.

\section{Conclusion}

Big Data technologies have greatly influenced the large organizations, which collectively undertake various types of large projects. This research is based on an online survey and interviews from management workforce; that are part of IT/Communication systems within enterprises. It represents analysis of different factors that are changing the rate of adoption of Big Data Technologies for communication management in large projects. The results show that fear of losing job is considered as a dominant factor by communication management workforce. On the basis of findings it can be concluded that many organizations still do not perceive the actual dynamism of Big Data Technologies and there is a need of proper training and ideas promotion. The element of Information Mobility was found as the leading factor contributing positively towards adoption of Big Data among the organizations. The findings of this research may equip the decision makers, the regulation agencies, and other driving bodies with related facts and findings about the adoption of big data technologies for communication management in large projects.

\section{References}

[1] S. L. Valle, "Big data, analytics and the path from insights to value", MIT sloan management review, vol. 52, no. 2, (2011), p. 21.

[2] D. Barton and D. Court, "Making advanced analytics work for you", Harvard business review, vol. 90, no. 10, (2012), pp. 78-83.

[3] H. Chen, R. H. L. Chiang and V. C. Storey, "Business Intelligence and Analytics: From Big Data to Big Impact", MIS quarterly, vol. 36, no. 4, (2012), pp. 1165-1188.

[4] R. E. Levitt, "Towards project management 2.0”, Engineering Project Organization Journal, vol. 1, no. 3, (2011), pp. 197-210.

[5] A. Davies and M. Hobday, "The Business of Projects: Managing Innovation in Complex Products and Systems", (2005).

[6] A. Davies and I. Mackenzie, "Project complexity and systems integration: Constructing the London 2012 Olympics and Paralympics Games", International Journal of Project Management, vol. 32, no. 5, (2014). pp. 773-790.

[7] D. L. Constantiou and J. Kallinikos, "New games, new rules: big data and the changing context of strategy", Journal of Information Technology, vol. 30, no. 1, (2015), pp. 44-57.

[8] S. Emmitt, "Managing interdisciplinary projects: a primer for architecture, engineering and construction", Routledge, (2010).

[9] S. Olga and Kurbel, "How to Prevent before You Must Cure-A Comprehensive Literature Review on Conflict Management Strategies in Global Project Teams", International Research Workshop on IT Project Management, (2012).

[10] D. J. Herbsleb and A. Mockus, "An empirical study of speed and communication in globally distributed software development”, Software Engineering, IEEE Transactions, vol. 29, no. 6, (2003), pp. 481-494.

[11] A. Ornäs, M. P. N. Hiort, P. Hynninen and C. L. T. N. A. Piri, "Practical Guide To Managing Distributed Software Development Projects", (2010).

[12] B. Bruegge, A. H. Dutoit and Timo Wolf, "Sysiphus: Enabling informal collaboration in global software development”, Global Software Engineering, 2006. ICGSE'06, International Conference on. IEEE, (2006).

[13] E. L. Cheng, "Face-to-face versus computer-mediated communication: Exploring employees' preference of effective employee communication channel", International journal for the advancement of science and arts, vol. 1, no. 2, (2010), pp. 38-47. 
[14] E. R. Rice, "Computer- mediated communication and organizational innovation", Journal of communication, vol. 37, no. 4, (1987), pp. 65-94.

[15] S. Arthur and S. M. Sheffrin, "Economics: Principles in action. Upper Saddle River, New Jersey 07458: Pearson Prentice Hall", (2003), p. 57

[16] Q. W. Meeker and Y. Hong, "Reliability meets big data: opportunities and challenges", Quality Engineering, vol. 26, no.1, (2014), pp. 102-116.
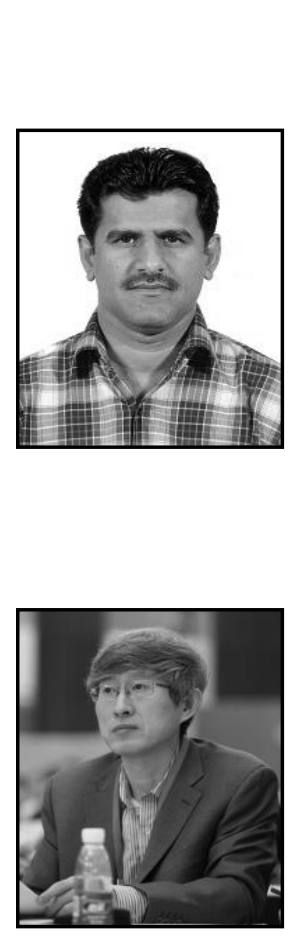

\section{Authors}

Suhail Memon, he is a Ph.D. researcher at Beijing University of Posts and Telecommunications, China. He has been affiliated to the education department since 2008. Before commencing his doctoral research, he worked as Teacher at Government High School in Pakistan. In $2005 \mathrm{He}$ received BCS (Hons.) from Quaid-e-Awam University of Engineering Science \& Technology Nawabshah Sindh, Pakistan. He received MBA (MIS) from University of Sindh Pakistan in 2008. His areas of research are Communication Management, Project Management, and Managing Information Systems.

Wang ChangFeng, he is the director of the International Project Management Institute in Beijing University of Posts and Telecommunications, professor, and Ph.D. supervisor. He is an expert in guiding the PMI GAC project management accreditation, assessment expert for PMI GAC CRC project management accreditation. His main research areas include the enterprise project management, safety risk early warning and emergency response, the complex system integration, and control of major projects and programs.

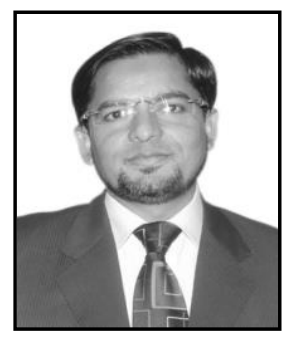

Shahid Rasheed, he is an experienced telecommunication management professional and an academic researcher at Beijing University of Posts and Telecommunications, China. His lengthiest association has been with Pakistan Telecommunication Company Ltd. (Etisalat), where he served in senior management roles mainly in the projects, programs, and strategic management functions. In addition to a doctorate degree in Management Science and Engineering domain, he holds post-graduation qualifications in Business Administration as well as Telecom Engineering fields. His areas of interest include Program and Strategy Management, ICT developments, and allied disciplines.

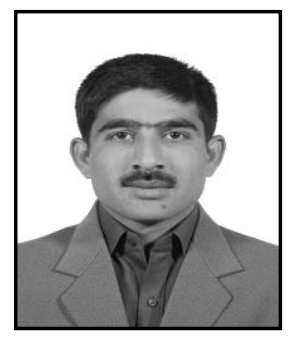

Zulfiqar Hussain Pathan, he is a Ph.D. scholar at Beijing University of Posts and Telecommunications, China. He has been affiliated to Mehran University of Engineering \& Technology, (MUET) Jamshoro, Pakistan. He got his post-graduation in the field of Technology \& Innovation Management and graduation in Computer Science. His research interests are Information System, Innovative \& Technological practices in Management in Public \& Private Sectors, and Entrepreneurship. 

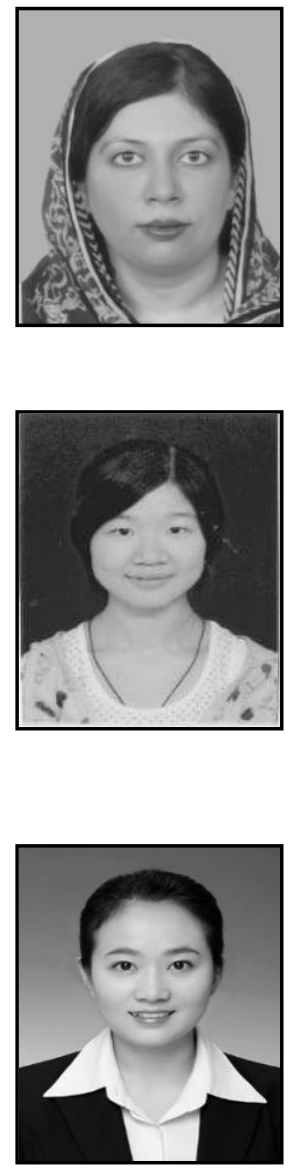

Sehrish Khan Saddozai, she is a Ph.D. researcher at Beijing University of Posts and Telecommunications, China in the school of Economics and Management. She received her Post graduation degree in sociology from Bahauddin Zakariya University Multan, Pakistan and also worked as a lecturer in Bahaudin Zakariya University Multan Pakistan. Her research interests are HRM, Talent Management and Project management.

Qiu Yixin, she is a Master researcher at Beijing University of Posts and Telecommunications, China. She has been affiliated to the Education since 2014. Before commencing her master's research, she studied in Jiangxi University of Science and Technology during 2010 to 2014. In 2014 she received bachelor's degree from Jiangxi University of Science and Technology. During her bachelor education, she won National Scholarship, Top Ten Graduate, Provincial "triple-A" outstanding student, the second award of national mathematics competition and some other prize.

Liu Yanping, she is a Master researcher at Beijing University of Posts and Telecommunications, She majored in Management Science and Engineering, Her current area of research is Project Management. She is the president of Project Management Innovation Institution Beijing University of Posts and Telecommunications. 
International Journal of Future Generation Communication and Networking Vol. 9, No.10, (2016) 